\title{
Roles for miRNAs in osteogenic differentiation of bone marrow mesenchymal stem cells
}

\author{
Jicheng Wang ${ }^{1,2}$, Shizhang Liu', Jingyuan $\mathrm{Li}^{1}$, Song Zhao ${ }^{1,2}$ and Zhi Yi ${ }^{1 *}$ (D
}

\begin{abstract}
Bone marrow mesenchymal stem cells (BMSCs), which were first discovered in bone marrow, are capable of differentiating into osteoblasts, chondrocytes, fat cells, and even myoblasts, and are considered multipotent cells. As a result of their potential for multipotential differentiation, self-renewal, immune regulation, and other effects, BMSCs have become an important source of seed cells for gene therapy, tissue engineering, cell replacement therapy, and regenerative medicine. MicroRNA (miRNA) is a highly conserved type of endogenous non-protein-encoding RNA of about 19-25 nucleotides in length, whose transcription process is independent of other genes. Generally, miRNA plays roles in regulating cell proliferation, differentiation, apoptosis, and development by binding to the $3^{\prime}$ untranslated region of target mRNAs, whereby they can degrade or induce translational silencing. Although miRNAs play a regulatory role in various metabolic processes, they are not translated into proteins. Several studies have shown that miRNAs play an important role in the osteogenic differentiation of BMSCs. Herein, we describe in-depth studies of roles for miRNAs during the osteogenic differentiation of BMSCs, as they provide new theoretical and experimental rationales for bone tissue engineering and clinical treatment.
\end{abstract}

Keywords: Bone marrow mesenchymal stem cells (BMSCs), MicroRNAs (miRNA), Osteogenic differentiation, Bone defects, Bone regeneration, Bone tissue engineering, Treatment

\section{Background}

Bone marrow mesenchymal stem cells (BMSCs), which were first discovered in bone marrow by Friedenstein et al. [1], can differentiate into aggregates similar to small-area bone or cartilage. After years of research, it was found that these cells can differentiate into osteoblasts, chondrocytes, adipocytes, and even myoblasts; thus, they are considered multi-potential cells. Indeed, as a result of their potential for multi-directional differentiation, self-renewal, immune regulation, and other effects, BMSCs have become an important source of seed cells in gene therapy, tissue engineering, cell replacement therapy, and regenerative medicine.

MicroRNA (miRNA), a highly conserved type of endogenous non-protein-encoding RNA about 19-25 nucleotides (nt) in length [2], can degrade or induce

\footnotetext{
*Correspondence: yizhi_7337@163.com

'Shaanxi Provincial People's Hospital, 256 Youyi West Road, Beilin, Xi'an

710068, China

Full list of author information is available at the end of the article
}

translational silence by binding to the 3 '-untranslated region ( $3^{\prime}$-UTR) of target mRNAs, thus affecting cell proliferation, differentiation, apoptosis, and ontogeny [3]. Transcription of miRNAs involves a highly conserved process that occurs independently of other genes. Notably, although miRNAs have been implicated in many metabolic processes, they are not translated into proteins [4]. Several studies have shown that miRNAs play an important role in the osteogenic differentiation of BMSCs. We describe in-depth studies of roles for miRNAs during osteogenic differentiation of BMSCs, as they provide new theoretical and experimental bases for bone tissue engineering and clinical treatment.

\section{Generation and biological function of miRNAs}

In 1993, Lee et al. [5] first discovered the presence of small RNAs in Caenorhabditis elegans and observed that they controlled biological processes such as the regulation of gene expression [6-8]. Around the same time, Wightman et al. reported the existence of small RNAs 
such as lin-4 [9]. Reinhart et al. found another small RNA with post-transcriptional regulation in the C. elegans: let-7 [10]. With deeper research, more than 1000 miRNAs have since been discovered, each of which regulates multiple mRNAs and is involved in the regulation of biological processes [11].

The production of miRNAs is a very complex biological process that includes two parts, nuclear synthesis and cytoplasmic synthesis, and requires the participation of a variety of enzymes. First, the gene encoding the miRNA is transcribed into a pri-miRNA with special hairpin structures (AAAAA and 7MGpppG) by RNA polymerase II within the nucleus. Next, pri-miRNAs are microcleaved by the nuclease Drosha (ribonuclease III) and processed into miRNA precursors of $70-80 \mathrm{nt}$ with a stem ring structure, i.e., pre-miRNAs. Exportin-5, a cytoplasmic transporter, transports pre-miRNAs from the nucleus into the cytoplasm with the assistance of Ran-GTP, and then the pre-miRNA is cleaved by ribonuclease III (Dicerase) into a duplex structure comprising an miRNA and miRNA* of about 19-23 nt. A miRNA* is the non-miRNA strand of a miRNA duplex generated by a Dicer-like enzyme from the miRNA stem-loop precursor; typically, miRNA*s are degraded. miRNAs form mature miRNAs by binding to argonaute proteins. Subsequently, the guide strand miRNA participates in miRNA transcription, while the passenger strand miRNA is degraded [12]. There are two manners by which mature miRNAs can form an RNA-induced silencing complex (RISC): (1) when the miRNA and target gene are fully complementary, the miRNA degrades the target gene; or (2) when the miRNA and target gene are not fully complementary, the combination of miRNA and 3'UTR inhibits translation of the target gene [13]. miRNAs are involved in several physiological processes, such as development, proliferation, differentiation, and apoptosis of normal cells, as well as in the maintenance of cellular pluripotency $[7,14]$.

\section{miRNAs in osteogenic differentiation of BMSCs}

Osteoblasts, which are involved in bone formation, are differentiated in vivo from BMSCs. Many studies have shown that miRNAs play an important role in osteogenic differentiation of BMSCs, as abnormal miRNA expression had important influences on their osteogenic differentiation $[15,16]$. Oskowitz et al. [17] found that after knocking out the mouse endonuclease Dicer, its BMSCs lost osteogenic differentiation. Dicer is an essential endonuclease for miRNA synthesis, indicating that miRNAs are closely related to bone formation and development [18]. Twenty-two differentially expressed miRNAs were identified in bone marrow mesenchymal stem cells (BMSCs) from patients with steroid-induced femoral head necrosis, 17 were upregulated and five were downregulated. During osteogenic differentiation of BMSCs, hsa-mir-601, hsa-mir-452-3p, hsa-mir-647, hsa-mir-516b-5p, and hsa-mir-127-5p were significantly downregulated, while hsa-mir-122-3p was significantly upregulated, suggesting that different miRNAs promoted or inhibited osteogenic differentiation [19]. miRNAs and their identified target genes in the osteogenic differentiation of BMSCs are summarized in Fig. 1.

\section{Anti-osteogenic differentiation miRNAs}

Tang et al. observed a time-dependent decrease in miR-124 expression during osteogenic differentiation of BMSCs, while alkaline phosphatase (ALP) activity and expression of osteocalcin $(\mathrm{OCN})$, osterix (Sp7), and runt-related transcription factor 2 (Runx2) were significantly increased. miR-124 overexpression inhibited osteogenic differentiation of BMSCs, and Sp7 was found to be a direct target [20]. miR-23a was significantly downregulated during osteogenic differentiation of BMSCs on the surface of titanium nanostructures, and its overexpression inhibited osteogenic differentiation of BMSCs; it was revealed that CXC chemokine ligand-12 (CXCL12) was a direct target of miR23a [21]. miR-23a-5p, another inhibitor, was also significantly downregulated during the osteogenic differentiation of BMSCs; however, upregulation of miR-23a-5p inhibited osteogenic differentiation of hBMSCs by directly targeting mitogen-activated protein kinase 13 (MAPK13) [22]. Similarly, miR-23a was found to be significantly downregulated during osteogenic differentiation of hBMSCs, and its overexpression directly targeted low-density lipoprotein receptor-associated protein 5 (LRP5) to inhibit osteogenic differentiation of hBMSCs [23]. Tian et al. found that overexpressed miR-23a bound to the 3'UTR of Runx2, which reduced its expression level and inhibited osteogenic differentiation of BMSCs. Notably, CXCL13 was found to attenuate the interaction between miRNA-23a and Runx2-3' UTR to promote osteogenic differentiation of BMSCs; thus, it can be used as a novel factor to promote osteogenic differentiation of BMSCs [24]. Deng et al. observed that miR$23 \mathrm{~b}$ overexpression significantly reduced Runx 2 expression levels during osteogenesis by directly binding to the 3 ' UTR of Runx2 and participating in tumor necrosis factor- $\alpha$-mediated osteogenic induction of BMSCs. Moreover, injection of Ad-Runx2 attenuated bone loss caused by miR-23b, thus providing a new direction for the treatment of osteoporosis [25].

Several studies have shown that miR-214 is significantly downregulated during osteogenic induction. First, overexpressed miR-214 was shown to bind to the 3'UTR of bone morphogenetic protein 2 (BMP2) to inhibit its expression; whereas, KCNQ1OT1 upregulated BMP2 expression by inhibiting miR-214 to promote osteogenic differentiation of BMSCs [26]. Subsequently, Guo et al. found that overexpression of miR-214 reduced ALP activity and gene expression of OCN, type I collagen $(\mathrm{Col}$ 


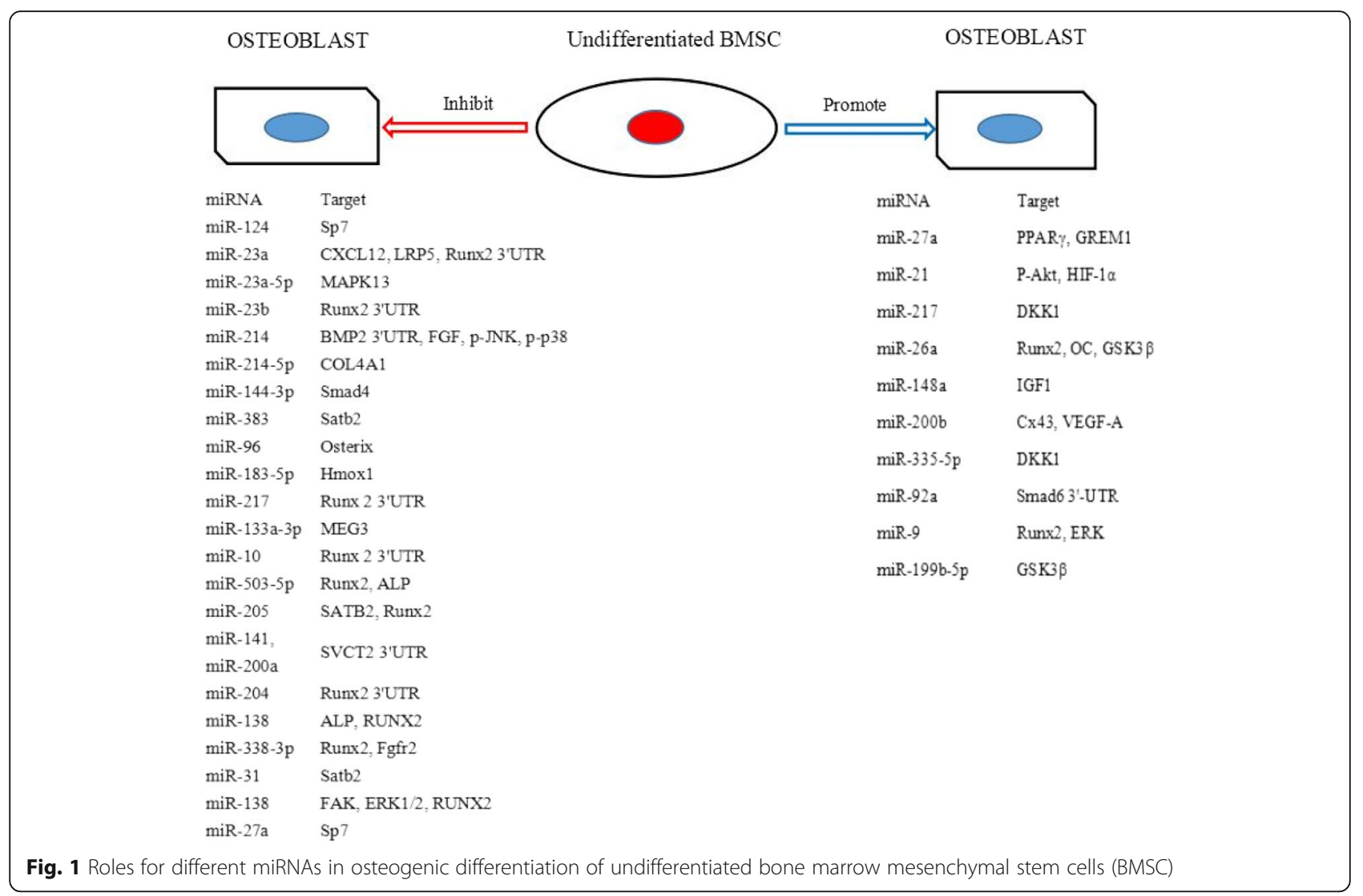

I), and osteopontin (OPN), thus inhibiting osteoblast differentiation of BMSCs. miR-214 overexpression was also shown to inhibit protein expression of fibroblast growth factor (FGF), phosphorylated c-Jun N-terminal kinase (p-JNK), and phosphorylated p38 mitogen-activated protein kinase (p-p38) in BMSCs, indicating that miR-214 inhibits osteogenic differentiation of BMSCs by inhibiting JNK and p38 pathways [27]. A later study showed that miR-214-5p inhibited expression of ALP, OCN, Runx2, and collagen alpha-1 (I) chain (COL1A1) in BMSCs during osteogenic differentiation, as well as transforming growth factor-beta (TGF- $\beta$ ), phosphorylated Smad2 (pSmad2), and collagen IV alpha-1 chain (COL4A1) proteins in BMSCs. Collectively, these results demonstrated that miR-214-5p may attenuate the osteogenic differentiation of BMSCs by regulating COL4A1 [28]. Moreover, the regulation of TGF- $\beta / \mathrm{Smad} 2 / \mathrm{COL} 4 \mathrm{~A} 1$ signaling to promote osteogenic differentiation of BMSCs is of great significance for the development of new treatments for postmenopausal osteoporosis.

Lu et al. reported that miR-144-3p inhibits osteogenic differentiation of BMSCs by reducing Smad4 expression [29]. Recent studies found that miR-383 inhibits osteogenic differentiation of rat BMSCs by targeting AT-rich sequencebinding protein 2 (Satb2) [30]. Osterix, a zinc fingercontaining transcription factor that plays an important role in osteogenic differentiation and bone formation [31], is also a downstream factor of Runx2, which is a necessary transcription factor for osteogenic differentiation, matrix production, and mineralization during bone formation [32]. Liu et al. observed that miR-96 is significantly upregulated in the serum of elderly patients with osteoporosis, and its overexpression reduced osteogenic differentiation of BMSCs by targeting osterix. Thus, inhibiting miRNA96 expression can increase osteogenic differentiation of BMSCs, making miR-96 a potential diagnostic marker or therapeutic target for age-related bone loss [33]. Studies have shown that miR-183-5p reduces proliferation and osteogenic differentiation of BMSCs by targeting heme oxygenase-1 (Hmox1), which accelerates aging [34]. Zhu et al. found that miR-217 targeting of the 3'UTR of Runx2 inhibited osteogenic differentiation of BMSCs through extracellular signal-regulated kinase (ERK) and p38 signaling pathways [35]. Upregulation of miR-133a-3p was observed in individuals with postmenopausal osteoporosis, in whom inhibition of miR-133a-3p was subsequently shown to promote osteogenic differentiation of BMSCs and improve symptoms [36]. miR-10 was also shown to inhibit osteogenic differentiation of BMSCs by targeting Runx2 and ERK pathways [37]. Liu et al. found that miR503-5p overexpression reduced protein expression levels of Runx 2 and ALP and inhibited osteogenic differentiation 
of BMSCs [38]. A summary of how these pathways are involved in the transition of BMSCs into osteocyte cells in shown in Fig. 2.

Hu et al. found that miR-205 binds to Satb2 and Runx2 via ERK and p38 MAPK signaling pathways, whereby it inhibits osteogenic differentiation of BMSCs [39]. Vitamin $\mathrm{C}$ is an essential nutrient for bone marrow stromal cell differentiation, collagen synthesis, and bone formation [40-45]. Notably, vitamin C is highly water soluble, but cannot enter the cell by simply diffusing through the lipid bilayer of the plasma membrane; instead, its transport must be mediated by a specific transporter. In BMSCs, vitamin $C$ is transported into cells via sodium-dependent vitamin C transporter 2 (SVCT2) [46]. miR-141 and miR200a inhibit SVCT2 expression by targeting its 3'UTR, thereby reducing osteogenic differentiation of BMSCs [47]. Zhao et al. showed that overexpressed miR-204 directly bound to the 3'UTR of Runx2, which promoted adipogenic differentiation and inhibited osteogenic differentiation; in contrast, downregulation of miR-204 increased osteogenesis and weakened the adipogenicity of BMSCs [48]. miR-138 was shown to inhibit osteogenic differentiation of BMSCs by targeting ALP and RUNX2. Moreover, delivery of anti-miR-138 to rats significantly enhanced the osteogenic capacity of BMSCs, indicating potentially important clinical significance of anti-miRNA138 for repair and regeneration of bone defects [49]. Liu et al. showed that overexpression of miR-338-3p can inhibit the expression of osterix by directly targeting Runx 2 and Fgfr2, thereby reducing osteoblast differentiation of BMSCs [50]. Deng et al. showed that overexpression of miR-31 could inhibit osteogenic differentiation of BMSCs, while downregulation of miR-31 significantly increased gene and protein expression levels of osteogenic-specific genes in vitro. In addition, injection of anti-miR-31 into rats enhanced the osteogenic differentiation potential and bone-regenerative ability of BMSCs [51]. A subsequent study confirmed that overexpression of miR-31 reduced Satb2 protein expression levels, which attenuated osteogenic differentiation of BMSCs, while downregulation of miR-31 promoted their osteogenic differentiation [52]. In addition, overexpression of miR-138 targets FAK, ERK1/2, and RUNX2 to inhibit osteogenic differentiation of BM SCs [53], which was confirmed in a second study that showed downregulation of miR-138 promotes mechanical tension-induced osteogenesis of hBMSCs [54]. Gong et al. found that overexpression of miR-27a significantly inhibited expression of Sp7, a target gene of miR-27a, and attenuated Satb2-induced osteogenic differentiation of BMSCs [55]. However, a later study found that upregulated miR-27a directly targets peroxisome proliferator-activated receptor gamma (PPAR $\gamma$ ) and gremlin 1 (GREM1), which inhibited lipogenesis and promoted osteogenic differentiation of BMSCs [56]. These results indicate that miR-27a can either promote or inhibit osteogenic differentiation of BMSCs by affecting different targets. miRNAs with anti-osteogenic differentiation effects are summarized in Table 1.

\section{Pro-osteogenic differentiation miRNAs}

Although many miRNAs inhibit osteogenic differentiation of BMSCs, exciting results from recent research have revealed miRNAs that promote osteogenic differentiation of BMSCs, including miR-21, miR-217, miR-26a, miR-148a, miR-200b, miR-335-5p, miR-92a, miR-9, and miR-199b-5p.

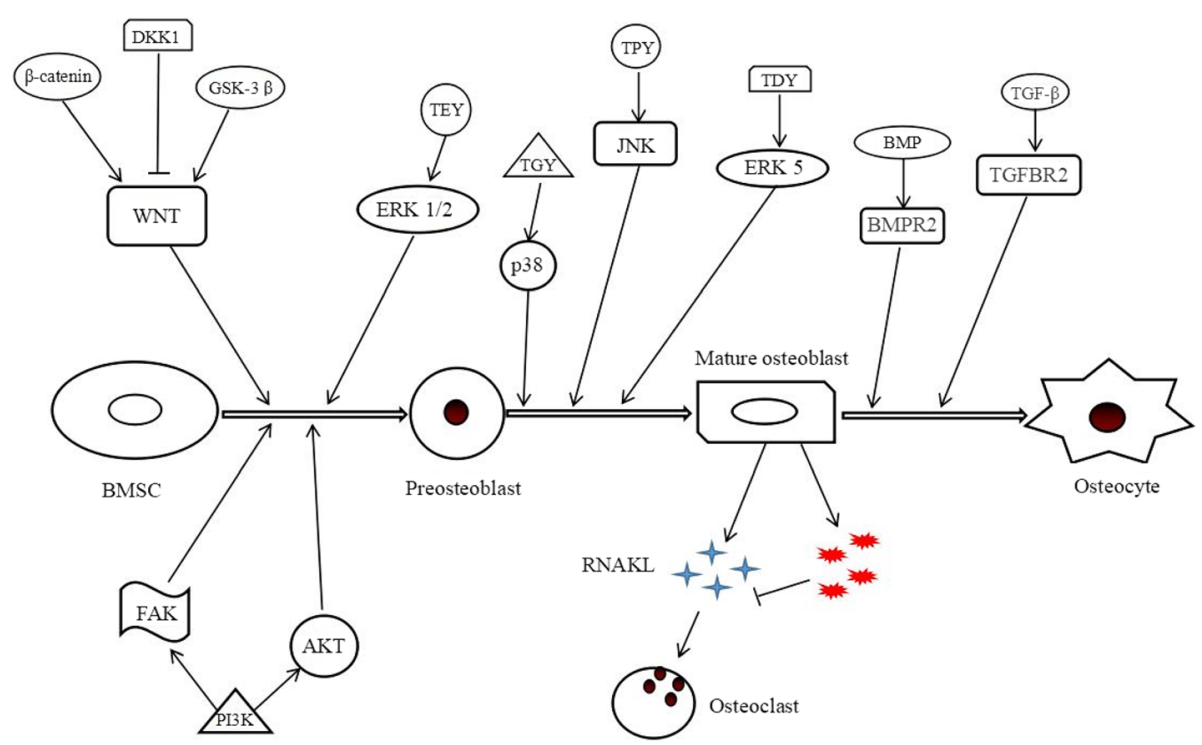

Fig. 2 Schematic representation of signaling pathways involved from bone mesenchymal stem cell to osteocyte cell 
Table 1 Anti-osteogenic differentiation miRNAs

\begin{tabular}{|c|c|c|c|}
\hline miRNAs & Target gene/target protein & Expression level & References \\
\hline miR-124 & Sp7 & $\uparrow$ & {$[20]$} \\
\hline miR-23a & CXCL12, LRP5, Runx2 3'UTR & $\uparrow$ & {$[21,23,24]$} \\
\hline miR-23a-5p & MAPK13 & $\uparrow$ & {$[22]$} \\
\hline miR-23b & Runx2 3'UTR & $\uparrow$ & {$[25]$} \\
\hline $\operatorname{miR}-214$ & BMP2 3'UTR, FGF, p-JNK, p-p38 & $\uparrow$ & {$[26,27]$} \\
\hline miR-214-5p & COL4A1 & $\uparrow$ & {$[28]$} \\
\hline miR-144-3p & Smad4 & $\uparrow$ & [29] \\
\hline miR-383 & Satb2 & $\uparrow$ & {$[30]$} \\
\hline miR-96 & Osterix & $\uparrow$ & [33] \\
\hline miR-183-5p & Hmox1 & $\uparrow$ & [34] \\
\hline miR-217 & Runx $23^{\prime}$ UTR & $\uparrow$ & {$[35]$} \\
\hline miR-133a-3p & MEG3 & $\uparrow$ & {$[36]$} \\
\hline miR-10 & Runx $23^{\prime}$ UTR & $\uparrow$ & {$[37]$} \\
\hline miR-503-5p & Runx2, ALP & $\uparrow$ & {$[38]$} \\
\hline miR-205 & SATB2, Runx2 & $\uparrow$ & {$[39]$} \\
\hline miR-141, miR-200a & SVCT2 3'UTR & $\uparrow$ & {$[47]$} \\
\hline miR-204 & Runx2 3'UTR & $\uparrow$ & [48] \\
\hline miR-138 & ALP, RUNX2 & $\uparrow$ & [49] \\
\hline miR-338-3p & Runx2, Fgfr2 & $\uparrow$ & {$[50]$} \\
\hline miR-31 & Satb2 & $\uparrow$ & {$[52]$} \\
\hline miR-138 & FAK, ERK1/2, RUNX2 & $\uparrow$ & [53] \\
\hline miR-27a & Sp7 & $\uparrow$ & {$[55]$} \\
\hline
\end{tabular}

Yang et al. showed that miR-21 promoted migration and osteogenic differentiation of BMSCs in vitro, as well as their osteogenic capacity, by increasing p-Akt and activating HIF-1 $\alpha$. Moreover, this study confirmed that the BMSC $/ \beta$-tricalcium phosphate ( $\beta$-TCP) complex modified by miRNA-21 had a significant osteogenic effect in repairing critical size defects [57]. Another study found that miR-21 was downregulated in bone tissue and serum of patients with osteoporosis. Expression of mineralized nodules and osteogenic genes was significantly increased in SD rats transfected with miR-21 analogues, indicating that miR-21 could promote osteogenic differentiation of BMSCs and, thus, providing a theoretical basis for the development of anti-osteoporosis drugs [58]. Dickkopf-1 (DKK1) is a $29-\mathrm{kDa}$ glycoprotein that plays an important role in maintaining bone metabolism and homeostasis. Abnormal expression of DKK1 is associated with a variety of bone-related diseases, while its inhibition promotes osteoblast differentiation and bone healing $[59,60]$. Dai et al. showed that miR-217 promotes nuclear translocation of $\beta$-catenin by targeting DKK1, which increases the expression of RUNX2 and COL1A1 to significantly promote proliferation and osteogenic differentiation of BMSCs [61]. This study provides a new approach to the treatment of steroid-associated osteonecrosis.
Repair of bone defects continues to be a major challenge for clinicians because it is difficult to restore bone function and regenerate bone loss. Implantation of BMSCs transfected with miR-26a and $\beta$-tricalcium phosphate biomaterials into the defect area of a mouse skull defect model increased bone regeneration and new bone volume, as well as gene and protein expression levels of Runx2 and OCN [62]. Studies have shown that miR-26a targets glycogen synthase kinase three beta (GSK3 $\beta$ ) to activate Wnt signaling and promote osteogenic differentiation of BMSCs [63]. Liu et al. found that low expression of miR-148a induces osteogenic differentiation of rat BMSCs by targeting insulin-like growth factor 1 (IGF1), and promotes fracture healing [64]. Subsequently, it was observed that rat BMSCs and human umbilical vein endothelial cells (HUVECs) are functionally connected by connexin 43 (Cx43), and miR-200b can be transferred from BMSCs to HUVECs via Cx43 to regulate osteogenesis and angiogenesis. Moreover, low expression of miR-200b inhibited VEGF-A expression, promoted osteogenic differentiation of BMSCs, and facilitated bone regeneration [65]. Zhang et al. found that overexpression of miR-335-5p induced osteogenic differentiation and bone formation of mouse BMSCs by targeting DKK1, and the resulting modified BMSCs have 
potential clinical therapeutic value for craniofacial bone regeneration [66]. Another study confirmed that a miR335-5p lipidoid-miRNA preparation (LMF-335) successfully delivered miR-335-5p into cells and promoted in vitro osteogenesis and in vivo skull bone healing, indicating that lipid-like miRNA delivery can be used to induce osteogenic differentiation of and bone regeneration by BMSCs [67]. Yan et al. reported that miR-92a targeted the 3'-UTR of Smad6 to inhibit Smad6-mediated Runx2 degradation and promote osteogenic differentiation of BMSCs [68]. A later study reported upregulation of miR-9 during osteogenic differentiation of BMSCs, as well as enhancement of their osteogenic capacity by its overexpression, and promotion of osteogenic differentiation through actions on Runx2 and ERK pathways [37]. Similarly, Zhao et al. showed that miR-199b-5p was significantly upregulated during osteogenesis, while its overexpression promoted osteoblast differentiation BMSCs through the GSK3 $\beta / \beta$-catenin signaling pathway [69]. miRNAs with pro-osteogenic effects are summarized in Table 2.

\section{Conclusion}

Bone marrow mesenchymal stem cells (BMSCs) are an important source of osteogenic seed cells in tissue engineering, and have good prospects for applications in the field of bone defect repair and regeneration. miRNAs play a key role in osteogenic differentiation of BMSCs, but their specific mechanisms of action are not fully understood. Indeed, as different miRNAs promote or inhibit osteogenic differentiation of BMSCs by affecting different targets, further study of the functional specificity of miRNA target genes and interactions between miRNAs is of great significance to elucidate their mechanisms of action. Gene therapy targeting miRNA target genes will benefit patients as research progresses. Moreover, with continuous development of biomedicine, molecular mechanisms underlying osteoblastic differentiation of BMSCs will be increasingly clarified,

Table 2 Pro-osteogenic differentiation miRNAs

\begin{tabular}{llll}
\hline miRNAs & Target gene/target protein & Expression level & References \\
\hline miR-27a & PPARY, GREM1 & $\uparrow$ & {$[56]$} \\
miR-21 & P-Akt, HIF-1a & $\uparrow$ & {$[57]$} \\
miR-217 & DKK1 & $\uparrow$ & {$[61]$} \\
miR-26a & Runx2, OC, GSK3 3 & $\uparrow$ & {$[62,63]$} \\
miR-148a & IGF1 & $\downarrow$ & {$[64]$} \\
miR-200b & Cx43, VEGF-A & $\downarrow$ & {$[65]$} \\
miR-335-5p & DKK1 & $\uparrow$ & {$[66]$} \\
miR-92a & Smad6 3'-UTR & $\uparrow$ & {$[68]$} \\
miR-9 & Runx2, ERK & $\uparrow$ & {$[37]$} \\
miR-199b-5p & GSK3 $\beta$ & $\uparrow$ & {$[69]$} \\
\hline
\end{tabular}

thus providing new theoretical and experimental rationales for bone tissue engineering and clinical treatment.

\section{Abbreviations}

ALP: Alkaline phosphatase; BMP2: Bone morphogenetic protein 2; BMSCs: Bone marrow mesenchymal stem cells; Col I: Type I collagen; COL4A1: Collagen IV alpha-1 chain; CX43: Connexin 43; CXCL13: CXC chemokine ligand-13; DKK1: Dickkopf-1; ERK: Extracellular signal-regulated kinase; GREM1: Gremlin 1; GSK3ß: Glycogen Synthase Kinase 3ß; HIF-

1a: Hypoxia-inducible factor-1a; Hmox1: Heme oxygenase-1; HUVECs: Human umbilical vein endothelial cells; IGF1: Insulin-like growth factor 1;

LRP5: Lipoprotein (LDL)-receptor-associated protein 5; MAPK13: Mitogenactivated protein kinase 13; miRNA: MicroRNA; OC: Osteocalcin;

OCN: Osteocalcin; OP: Osteoporosis; OPN: Osteopontin; p38 MAPK: p38 mitogen-activated protein kinase; PMOP: Postmenopausal osteoporosis; PPARY: Peroxisome proliferator-activated receptor gamma; Runx2: Runtrelated transcription factor 2; Satb2: AT-rich sequence-binding protein 2; Smad4: Drosophila mothers against decapentaplegic 4; SONFH: Steroidinduced femoral head necrosis; Sp7: Osterix; SVCT2: Sodium-dependent vitamin C transporter 2; VEGF-A: Vascular epidermal growth factor-A

\section{Acknowledgements}

We thank Professor Zhi Yi for his careful guidance in the process of writing papers. Thank you for your understanding and support.

\section{Authors' contributions}

JW drafted the manuscript and revised the manuscript. SL and ZY contributed to manuscript conception. SL, SZ, and JL provided the financial support. All authors read and approved the final manuscript.

\section{Funding}

This work was supported by the Shaanxi Provincial Science and Technology Department (No. 2018ZDXM-SF-054)

Availability of data and materials

Not applicable.

Ethics approval and consent to participate

Not applicable.

Consent for publication

Not applicable.

Competing interests

The authors declare that they have no competing interests.

Author details

'Shaanxi Provincial People's Hospital, 256 Youyi West Road, Beilin, Xi'an 710068, China. ${ }^{2}$ Xi'an Medical University, Xi'an 710068, China.

Published online: 28 June 2019

\section{References}

1. Friedenstein AJ, Chailakhyan RK, Gerasimov UV. Bone marrow osteogenic stem cells: in vitro cultivation and transplantation in diffusion chambers. Cell Tissue Kinet. 1987;20(3):263-72.

2. Beyer C, Zampetaki A, Lin NY, Kleyer A, Perricone C, lagnocco A, Distler A, Langley SR, Gelse K, Sesselmann S, et al. Signature of circulating microRNAs in osteoarthritis. Ann Rheum Dis. 2015;74(3):e18. https://doi.org/10.1136/ annrheumdis-2013-204698.

3. Jackson RJ, Standart N. How do MicroRNAs regulate gene expression? Sci STKE. 2007;2007(367):re1. https://doi.org/10.1126/stke.3672007re1.

4. Ha M, Kim VN. Regulation of microRNA biogenesis. Nat Rev Mol Cell Biol. 2014;15(8):509-24. https://doi.org/10.1038/nrm3838.

5. Lee RC, Feinbaum RL, Ambros V. The C. elegans heterochronic gene lin-4 encodes small RNAs with antisense complementarity to lin-14. Cell. 1993; 75(5):843-54.

6. Hwang HW, Mendell JT. MicroRNAs in cell proliferation, cell death, and tumorigenesis. Br J Cancer. 2006;94(6):776-80. https://doi.org/10.1038/sj.bjc. 6603023. 
7. Ebert MS, Sharp PA. Roles for microRNAs in conferring robustness to biological processes. Cell. 2012;149(3):515-24. https://doi.org/10.1016/j.cell.2012.04.005.

8. Fire A, Xu S, Montgomery MK, Kostas SA, Driver SE, Mello CC. Potent and specific genetic interference by double-stranded RNA in Caenorhabditis elegans. Nature. 1998;391(6669):806-11. https://doi.org/10.1038/35888.

9. Wightman B, Ha I, Ruvkun G. Posttranscriptional regulation of the heterochronic gene lin-14 by lin- 4 mediates temporal pattern formation in C. elegans. Cell. 1993;75(5):855-62.

10. Reinhart BJ, Slack FJ, Basson M, Pasquinelli AE, Bettinger JC, Rougvie AE, Horvitz HR, Ruvkun G. The 21-nucleotide let-7 RNA regulates developmental timing in Caenorhabditis elegans. Nature. 2000;403(6772):901-6. https://doi. org/10.1038/35002607.

11. Farh KK, Grimson A, Jan C, Lewis BP, Johnston WK, Lim LP, Burge CB, Bartel DP. The widespread impact of mammalian MicroRNAs on mRNA repression and evolution. Science. 2005;310(5755):1817-21. https://doi.org/10.1126/ science.1121158.

12. Jones-Rhoades MW, Bartel DP, Bartel B. MicroRNAS and their regulatory roles in plants. Annu Rev Plant Biol. 2006;57:19-53. https://doi.org/10.1146/ annurev.arplant.57.032905.105218.

13. Huntzinger $E$, lzaurralde $E$. Gene silencing by microRNAs: contributions of translational repression and mRNA decay. Nat Rev Genet. 2011;12(2):99-110. https://doi.org/10.1038/nrg2936.

14. Kosik KS. The neuronal microRNA system. Nat Rev Neurosci. 2006;7(12):91120. https://doi.org/10.1038/nrn2037.

15. Kang $\mathrm{H}$, Hata A. The role of microRNAs in cell fate determination of mesenchymal stem cells:balancing adipogenesis and osteogenesis. BMB Rep. 2015;48(6):319-23.

16. Arfat Y, Xiao WZ, Ahmad M, Zhao F, Li DJ, Sun YL, Hu L, Zhihao C, Zhang G, Iftikhar $S$, et al. Role of microRNAs in osteoblasts differentiation and bone disorders. Curr Med Chem. 2015;22(6):748-58.

17. Oskowitz AZ, Lu J, Penfornis P, Ylostalo J, McBride J, Flemington EK, Prockop DJ, Pochampally R. Human multipotent stromal cells from bone marrow and microRNA: regulation of differentiation and leukemia inhibitory factor expression. Proc Natl Acad Sci U S A. 2008;105(47):18372-7. https://doi.org/ 10.1073/pnas.0809807105.

18. Harfe BD, McManus MT, Mansfield JH, Hornstein E, Tabin CJ. The RNasell enzyme dicer is required for morphogenesis but not patterning of the vertebrate limb. Proc Natl Acad Sci U S A. 2005;102(31):10898-903. https:// doi.org/10.1073/pnas.0504834102

19. Wang A, Ren M, Song Y, Wang X, Wang $Q$, Yang $Q$, Liu H, Du Z, Zhang G, Wang J. MicroRNA expression profiling of bone marrow mesenchymal stem cells in steroid-induced osteonecrosis of the femoral head associated with osteogenesis. Med Sci Monit. 2018;24:1813-25.

20. Tang JZ, Lin X, Zhong JY, Xu F, Wu F, Liao XB, Cui RR, Li F, Yuan LQ. miR124 regulates the osteogenic differentiation of bone marrow-derived mesenchymal stem cells by targeting Sp7. Mol Med Rep. 2019. https://doi. org/10.3892/mmr.2019.10054

21. Zhuang XM, Zhou B, Yuan KF. Role of p53 mediated miR-23a/CXCL12 pathway in osteogenic differentiation of bone mesenchymal stem cells on nanostructured titanium surfaces. Biomed Pharmacother. 2019;112:108649. https://doi.org/10.1016/j.biopha.2019.108649.

22. Ren G, Sun J, Li MM, Zhang YD, Li RH, Li YM. MicroRNA-23a-5p regulates osteogenic differentiation of human bone marrow-derived mesenchymal stem cells by targeting mitogen-activated protein kinase-13. Mol Med Rep. 2018;17(3):4554-60. https://doi.org/10.3892/mmr.2018.8452.

23. Li T, Li H, Wang Y, Li T, Fan J, Xiao K, Zhao RC, Weng X. microRNA-23a inhibits osteogenic differentiation of human bone marrow-derived mesenchymal stem cells by targeting LRP5. Int J Biochem Cell Biol. 2016;72: 55-62. https://doi.org/10.1016/j.biocel.2016.01.004.

24. Tian F, Ji XL, Xiao WA, Wang B, Wang F. CXCL13 promotes osteogenic differentiation of mesenchymal stem cells by inhibiting miR-23a expression. Stem Cells Int. 2015;2015:632305. https://doi.org/10.1155/2015/632305.

25. Deng L, Hu G, Jin L, Wang C, Niu H. Involvement of microRNA-23b in TNFa-reduced BMSC osteogenic differentiation via targeting runx2. J Bone Miner Metab. 2018;36(6):648-60. https://doi.org/10.1007/s00774-017-0886-8.

26. Wang CG, Liao Z, Xiao H, Liu H, Hu YH, Liao QD, Zhong D. LncRNA KCNQ1OT1 promoted BMP2 expression to regulate osteogenic differentiation by sponging miRNA-214. Exp Mol Pathol. 2019:107:77-84 https://doi.org/10.1016/j.yexmp.2019.01.012.

27. Guo Y, Li L, Gao J, Chen X, Sang Q. miR-214 suppresses the osteogenic differentiation of bone marrow-derived mesenchymal stem cells and these effects are mediated through the inhibition of the JNK and p38 pathways. Int J Mol Med. 2017;39(1):71-80. https://doi.org/10.3892/ijmm.2016.2826.

28. Qiu J, Huang G, Na N, Chen L. MicroRNA-214-5p/TGF- $\beta /$ Smad2 signaling alters adipogenic differentiation of bone marrow stem cells in postmenopausal osteoporosis. Mol Med Rep. 2018;17(5):6301-10. https://doi org/10.3892/mmr.2018.8713.

29. Lu J, Zhang H, Yu P, Gong Y, Gong X, Fan Q, Yang Y. Role of miR-144-3p and its target gene in regulating osteogenic differentiation of rat bone marrow mesenchymal stem cells in vitro. Nan Fang Yi Ke Da Xue Xue Bao. 2018;38(9):1083-8. https://doi.org/10.12122/j.issn.1673-4254.2018.09.10.

30. Tang J, Zhang Z, Jin X, Shi H. miR-383 negatively regulates osteoblastic differentiation of bone marrow mesenchymal stem cells in rats by targeting Satb2. Bone. 2018;114:137-43. https://doi.org/10.1016/j.bone.2018.06.010.

31. Nakamura T, Toita H, Yoshimoto A, Nishimura D, Takagi T, Ogawa T, Takeya T, Ishida-Kitagawa N. Potential involvement of Twist2 and Erk in the regulation of osteoblastogenesis by HB-EGF-EGFR signaling. Cell Struct Funct. 2010;35(1):53-61.

32. Nishio Y, Dong Y, Paris M, O'Keefe RJ, Schwarz EM, Drissi H. Runx2-mediated regulation of the zinc finger Osterix/Sp7 gene. Gene. 2006;372:62-70. https://doi.org/10.1016/j.gene.2005.12.022.

33. Liu H, Liu Q, Wu XP, He HB, Fu L. MiR-96 regulates bone metabolism by targeting osterix. Clin Exp Pharmacol Physiol. 2018;45(6):602-13. https://doi. org/10.1111/1440-1681.12912.

34. Davis C, Dukes A, Drewry M, Helwa I, Johnson MH, Isales CM, Hill WD, Liu Y, Shi X, Fulzele S, et al. MicroRNA-183-5p increases with age in bone-derived extracellular vesicles, suppresses bone marrow stromal (stem) cell proliferation, and induces stem cell senescence. Tissue Eng Part A. 2017; 23(21-22):1231-40. https://doi.org/10.1089/ten.TEA.2016.0525.

35. Zhu YL, Wang S, Ding DG, Xu L, Zhu HT. miR-217 inhibits osteogenic differentiation of rat bone marrow-derived mesenchymal stem cells by binding to Runx2. Mol Med Rep. 2017;15(5):3271-7. https://doi.org/10.3892/ mmr.2017.6349.

36. Wang $Q$, Li Y, Zhang Y, Ma L, Lin L, Meng J, Jiang L, Wang L, Zhou P, Zhang $Y$. LncRNA MEG3 inhibited osteogenic differentiation of bone marrow mesenchymal stem cells from postmenopausal osteoporosis by targeting miR-133a-3p. Biomed Pharmacother. 2017;89:1178-86. https://doi.org/10. 1016/j.biopha.2017.02.090.

37. Luo H, Gao H, Liu F, Qiu B. Regulation of Runx2 by microRNA-9 and microRNA-10 modulates the osteogenic differentiation of mesenchymal stem cells. Int J Mol Med. 2017;39(4):1046-52. https://doi.org/10.3892/ijmm. 2017.2918.

38. Liu L, Liu M, Li R, Liu H, Du L, Chen H, Zhang Y, Zhang S, Liu D. MicroRNA503-5p inhibits stretch-induced osteogenic differentiation and bone formation. Cell Biol Int. 2017;41(2):112-23. https://doi.org/10.1002/cbin. 10704.

39. Hu N, Feng C, Jiang Y, Miao Q, Liu H. Regulative effect of mir-205 on osteogenic differentiation of bone mesenchymal stem cells (BMSCs): possible role of SATB2/Runx2 and ERK/MAPK pathway. Int J Mol Sci. 2015; 16(5):10491-506. https://doi.org/10.3390/ijms160510491.

40. Bellows CG, Aubin JE, Heersche JN, Antosz ME. Mineralized bone nodules formed in vitro from enzymatically released rat calvaria cell populations. Calcif Tissue Int. 1986;38(3):143-54.

41. Choi KM, Seo YK, Yoon HH, Song KY, Kwon SY, Lee HS, Park JK. Effect of ascorbic acid on bone marrow derived mesenchymal stem cell proliferation and differentiation. J Biosci Bioeng. 2008;105(6):586-94. https://doi.org/10. 1263/jbb.105.586

42. Lee KL, Aubin JE, Heersche JN. Beta-Glycerophosphate-induced mineralization of osteoid does not alter expression of extracellular matrix components in fetal rat calvarial cell cultures. J Bone Miner Res. 1992;7(10): 1211-9. https://doi.org/10.1002/jbmr.5650071013.

43. Maehata Y, Takamizawa S, Ozawa S, Izukuri K, Kato Y, Sato S, Lee MC, Kimura A, Hata R. Type III collagen is essential for growth acceleration of human osteoblastic cells by ascorbic acid 2-phosphate, a long-acting vitamin C derivative. Matrix Biol. 2007;26(5):371-81. https://doi.org/10.1016/j.matbio. 2007.01.005

44. Urban K, Höhling HJ, Lüttenberg B, Szuwart T, Plate U; Biomineralisation Research Unit. An in vitro study of osteoblast vitality influenced by the vitamins C and E. Head Face Med 2012; 8:25. doi: https://doi.org/10.1186/ 1746-160X-8-25.

45. Wei F, Qu C, Song T, Ding G, Fan Z, Liu D, Liu Y, Zhang C, Shi S, Wang S. Vitamin $C$ treatment promotes mesenchymal stem cell sheet formation and 
tissue regeneration by elevating telomerase activity. J Cell Physiol. 2012; 227(9):3216-24. https://doi.org/10.1002/jcp.24012.

46. Fulzele S, Chothe P, Sangani R, Chutkan N, Hamrick M, Bhattacharyya M, Prasad PD, Zakhary I, Bowser M, Isales C, et al. Sodium-dependent vitamin C transporter SVCT2: expression and function in bone marrow stromal cells and in osteogenesis. Stem Cell Res. 2013;10(1):36-47. https://doi.org/10. 1016/j.scr.2012.08.004.

47. Sangani R, Periyasamy-Thandavan S, Kolhe R, Bhattacharyya MH, Chutkan N, Hunter M, Isales C, Hamrick M, Hill WD, Fulzele S. MicroRNAs-141 and 200a regulate the SVCT2 transporter in bone marrow stromal cells. Mol Cell Endocrinol. 2015;410:19-26. https://doi.org/10.1016/j.mce.2015.01.007.

48. Zhao J, Wang C, Song Y, Fang B. Arsenic trioxide and microRNA-204 display contrary effects on regulating adipogenic and osteogenic differentiation of mesenchymal stem cells in aplastic anemia. Acta Biochim Biophys Sin Shanghai. 2014;46(10):885-93. https://doi.org/10.1093/abbs/gmu082.

49. Yan J, Zhang C, Zhao Y, Cao C, Wu K, Zhao L, Zhang Y. Non-viral oligonucleotide antimiR-138 delivery to mesenchymal stem cell sheets and the effect on osteogenesis. Biomaterials. 2014;35(27):7734-49. https://doi. org/10.1016/j.biomaterials.2014.05.089.

50. Liu H, Sun Q, Wan C, Li L, Zhang L, Chen Z. MicroRNA-338-3p regulates osteogenic differentiation of mouse bone marrow stromal stem cells by targeting Runx2 and Fgfr2. J Cell Physiol. 2014;229(10):1494-502. https://doi. org/10.1002/jcp.24591.

51. Deng Y, Bi X, Zhou H, You Z, Wang Y, Gu P, Fan X. Repair of critical-sized bone defects with anti-miR-31-expressing bone marrow stromal stem cells and poly(glycerol sebacate) scaffolds. Eur Cell Mater. 2014;27:13-24; discussion 24-5.

52. Deng Y, Wu S, Zhou H, Bi X, Wang Y, Hu Y, Gu P, Fan X. Effects of a miR-31, RunX2, and Satb2 regulatory loop on the osteogenic differentiation of bone mesenchymal stem cells. Stem Cells Dev. 2013;22(16):2278-86. https://doi. org/10.1089/scd.2012.0686.

53. Hu J, Liao H, Ma Z, Chen H, Huang Z, Zhang Y, Yu M, Chen Y, Xu J. Focal adhesion kinase signaling mediated the enhancement of osteogenesis of human mesenchymal stem cells induced by extracorporeal shockwave. Sci Rep. 2016;6:20875. https://doi.org/10.1038/srep20875.

54. Wu J, Zhao J, Sun L, Pan Y, Wang H, Zhang WB. Long non-coding RNA H19 mediates mechanical tension-induced osteogenesis of bone marrow mesenchymal stem cells via FAK by sponging miR-138. Bone. 2018;108:6270. https://doi.org/10.1016/j.bone.2017.12.013.

55. Gong Y, Lu J, Yu X, Yu Y. Expression of Sp7 in Satb2-induced osteogenic differentiation of mouse bone marrow stromal cells is regulated by microRNA-27a. Mol Cell Biochem. 2016;417(1-2):7-16. https://doi.org/10. 1007/s11010-016-2709-y.

56. Gu C, Xu Y, Zhang S, Guan H, Song S, Wang X, Wang Y, Li Y, Zhao G. miR27 attenuates adipogenesis and promotes osteogenesis in steroid-induced rat BMSCs by targeting PPARY and GREM1. Sci Rep. 2016;6:38491. https:// doi.org/10.1038/srep38491.

57. Yang C, Liu X, Zhao K, Zhu Y, Hu B, Zhou Y, Wang M, Wu Y, Zhang C, Xu J. miRNA-21 promotes osteogenesis via the PTEN/PI3K/Akt/HIF-1a pathway and enhances bone regeneration in critical size defects. Stem Cell Res Ther. 2019;10(1):65. https://doi.org/10.1186/s13287-019-1168-2.

58. Zhao Z, Li X, Zou D, Lian Y, Tian S, Dou Z. Expression of microRNA-21 in osteoporotic patients and its involvement in the regulation of osteogenic differentiation. Exp Ther Med. 2019;17(1):709-14. https://doi.org/10.3892/ etm.2018.6998.

59. Zhang M, Bian $Y Q$, Tao HM, Yang XF, Mu WD. Simvastatin induces osteogenic differentiation of MSCs via Wnt/B-catenin pathway to promote fracture healing. Eur Rev Med Pharmacol Sci. 2018;22(9):2896-905. https:// doi.org/10.26355/eurrev_201805_14992.

60. Li B, Sun J, Dong Z, Xue P, He X, Liao L, Yuan L, Jin Y. GCN5 modulates osteogenic differentiation of periodontal ligament stem cells through DKK1 acetylation in inflammatory microenvironment. Sci Rep. 2016;6:26542. https://doi.org/10.1038/srep26542.

61. Dai Z, Jin Y, Zheng J, Liu K, Zhao J, Zhang S, Wu F, Sun Z. MiR-217 promotes cell proliferation and osteogenic differentiation of BMSCs by targeting DKK1 in steroid-associated osteonecrosis. Biomed Pharmacother. 2019;109:1112-9. https://doi.org/10.1016/j.biopha.2018.10.166.

62. Liu Z, Chang H, Hou Y, Wang Y, Zhou Z, Wang M, Huang Z, Yu B. Lentivirusmediated microRNA-26a overexpression in bone mesenchymal stem cells facilitates bone regeneration in bone defects of calvaria in mice. Mol Med Rep. 2018;18(6):5317-26. https://doi.org/10.3892/mmr.2018.9596.
63. Su X, Liao L, Shuai Y, Jing H, Liu S, Zhou H, Liu Y, Jin Y. MiR-26a functions oppositely in osteogenic differentiation of BMSCs and ADSCs depending on distinct activation and roles of Wnt and BMP signaling pathway. Cell Death Dis. 2015;6:e1851. https://doi.org/10.1038/cddis.2015.221.

64. Liu H, Su H, Wang X, Hao W. MiR-148a regulates bone marrow mesenchymal stem cells-mediated fracture healing by targeting insulin-like growth factor 1. J Cell Biochem. 2018. https://doi.org/10.1002/jcb.27121.

65. Fan $X$, Teng $Y, Y$ Y Z, Zhou Y, Tan WS. The effect of gap junction-mediated transfer of miR-200b on osteogenesis and angiogenesis in a co-culture of MSCs and HUVECs. J Cell Sci. 2018;131(13). https://doi.org/10.1242/jcs.216135.

66. Zhang L, Tang Y, Zhu X, Tu T, Sui L, Han Q, Yu L, Meng S, Zheng L, Valverde $P$, et al. Overexpression of MiR-335-5p promotes bone formation and regeneration in mice. J Bone Miner Res. 2017;32(12):2466-75. https://doi. org/10.1002/jbmr.3230

67. Sui L, Wang M, Han Q, Yu L, Zhang L, Zheng L, Lian J, Zhang J, Valverde P, $X u \mathrm{Q}$, et al. A novel Lipidoid-MicroRNA formulation promotes calvarial bone regeneration. Biomaterials. 2018;177:88-97. https://doi.org/10.1016/j. biomaterials.2018.05.038.

68. Yan X, Wang H, Li Y, Jiang Y, Shao Q, Xu W. MicroRNA-92a overexpression promotes the osteogenic differentiation of bone mesenchymal stem cells by impeding Smad6-mediated runt-related transcription factor 2 degradation. Mol Med Rep. 2018;17(6):7821-6. https://doi.org/10.3892/mmr. 2018.8829.

69. Zhao R, Li Y, Lin Z, Wan J, Xu C, Zeng Y, Zhu Y. miR-199b-5p modulates

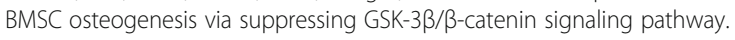
Biochem Biophys Res Commun. 2016;477(4):749-54. https://doi.org/10.1016/ j.bbrc.2016.06.130

\section{Publisher's Note}

Springer Nature remains neutral with regard to jurisdictional claims in published maps and institutional affiliations.
Ready to submit your research? Choose BMC and benefit from:

- fast, convenient online submission

- thorough peer review by experienced researchers in your field

- rapid publication on acceptance

- support for research data, including large and complex data types

- gold Open Access which fosters wider collaboration and increased citations

- maximum visibility for your research: over $100 \mathrm{M}$ website views per year

At BMC, research is always in progress.

Learn more biomedcentral.com/submissions 